\title{
Komponieren für das Radio: Akteure, Diskurse, Praktiken
}

Die Beiträge des Hauptsymposiums „Komponieren für das Radio: Akteure, Diskurse, Praktiken" unter der Leitung von Prof. Dr. Camilla Bork (Leuven) und Prof. Dr. Antje Tumat (Detmold/ Paderborn) sind in einem Band der Zeitschrift Musiktheorie im Juni 2020 erschienen. Im Folgenden werden die Einleitung und die Abstracts abgedruckt, für die Texte selbst, die größtenteils auf Deutsch verfasst sind, sei auf den Band Komponieren für das Radio, hrsg. von Camilla Bork und Antje Tumat (= Musiktheorie 2/2020), Laaber 2020, verwiesen.

\section{Einleitung}

Der vorliegende Band versammelt die Beiträge des interdisziplinären Hauptsymposiums „Komponieren für das Radio", welches im September 2019 im Rahmen der Jahrestagung der Gesellschaft für Musikforschung an der Universität Paderborn und der Hochschule für Musik Detmold stattfand. Ziel war es, den Einfluss des Mediums Radio auf den Kompositionsprozess von für das Radio geschriebenen Werken einerseits und die durch radiophone Kompositionen bzw. radiophonen Klang ausgelösten Diskurse andererseits zu untersuchen. Beides wurde anhand von internationalen Fallbeispielen in chronologischer Abfolge thematisiert: Ausgehend von Pionieren der Radiokunst in den 1920er und 1930er Jahren, deren Arbeiten oftmals jenseits aller Aufzeichnungen direkt im Studio produziert wurden, über Produktionen, welche die Technologie des Magnettonbands nutzend seit Ende der 1940er Jahre entstanden, bis hin zu Radioproduktionen der Gegenwart. Dabei lassen sich einige thematische Achsen hervorheben: Fast alle Fallbeispiele zeigen, dass in den Radiokompositionen immer auch selbstreferentiell die Bedingungen und Geschichte radiophonen Klangs mitkomponiert werden - sei es als Reflexion eines Mediums, das wie im Falle August Baeyens zu Beginn der 1920er Jahre aufs engste mit populärer Musik und einer neusachlichen Ästhetik verknüpft war, oder sei es als Erinnerung historischer Radiostimmen bzw. Stimmästhetiken und ihrer politischen Implikationen bei Mauricio Kagel und Bernd Alois Zimmermann. Kompositorisch reflektiert wird dabei auch die räumliche Dimension des Radios. Radioklang überbrückt nicht nur Räume und setzt sie miteinander in Beziehung (etwa in den Werken Elisabeth Schimanas), sondern lässt Raum und Entfernung im ,Rauschen des Äthers' erst hörbar werden (z. B. in John Cages Imaginary Landscape No. 4). Wie sehr dabei der Radioklang selbst - vor allem unter den Bedingungen des Digitalen und neuer Technologien - räumlichen Modulationen und Manipulationen unterworfen ist, macht ein musiksystematischer Ausblick am Ende des Heftes deutlich. Schließlich ist radiophones Komponieren aufs engste an Fragen von Macht und Identität geknüpft - und zwar von Beginn seiner Geschichte an, als das Radio 1923 als staatlich streng reguliertes Medium der Weimarer Republik zu senden begann, dessen dezidiert politischer Charakter gerade 
in seiner scheinbar apolitischen Fokussierung auf Kultur und Erziehung deutlich wird. Facetten dieser politischen Dimension durchziehen die Beispiele der 1950er und 1960er Jahre ebenso wie die zeitgenössische Radio Art, die die Hörerinnen und Hörer u. a. mit Fragen von Macht und Sound bzw. Hören im Blick auf gender- und transgender-Identitäten konfrontiert, wie z. B. im Werk Schimanas und Terre Thaemlitz'. Nicht in dem Band enthalten ist die Podiumsdiskussion über den Einfluss der Digitalisierung auf die Zukunft radiophoner Musik und Kunst mit Marcus Gammel (Deutschlandfunk Kultur), Prof. Dr. Wolfgang Hagen (Medienwissenschaftler) und Max Hundelshausen (Komponist).

Für die Finanzierung des Symposiums danken wir der Fritz Thyssen Stiftung sehr herzlich.

Leuven und Paderborn, im Juli 2020

Camilla Bork und Antje Tumat

\section{Abstracts der Beiträge}

\section{Nils Grosch: „,Der Rundfunk als Distributionsapparat': Eine Notiz zu Musik und Neuen Medien in der Weimarer Republik"}

Brecht's demand to transform the radio from a distribution device into a communication device challenges us to question radio theories and the radiophone concepts of the Weimar Republic for their basic communicative assumptions. What are the medial and distributive ideas of art? What cultural-political and communication-theoretical scope and consequences did the broadcasting contract policy mean? What does the call for broadcasting as a new patron mean and how does it fit into the idea of a democratic culture of the post-revolutionary period?

\section{Mark Delaere: „Ein Hörspiel aus der Pionierzeit des belgischen öffentlichen Rundfunks N.I.R.: August L. Baeyens' La Sonate d'Amour (1934)“}

This article offers an insight into the structure, organization and mission of the music department and its radio orchestras of the Belgian public broadcast N.I.R. at the time of its establishment in 1930. This overview forms the historic background for the 'radiophonic novel' La Sonate d'Amour (1934) based on a text by Roger Avermaete and with music by August L. Baeyens, the analysis of which being the principal aim of this article. The melodramatic plot 
is but a pretext for the real drama: the function of the modern composer in a society largely oriented towards the past. To that end, Baeyens compresses the evolution of music from Beethoven to Schoenberg, Hindemith and jazz music in his radio play.

\section{Andreas Meyer: „Random Radio. John Cages Imaginary Landscape No. 4 (1951) und die Poetik der ,indeterminacy'“}

Since the 1950s, John Cage's impact on public perception has been particularly linked to radio: from the legendary 'theatrical pieces' with radio receivers on stage (Water Music, Water Walk) to the late productions for the Westdeutscher Rundfunk. From about 1940, Cage experimented with the then new possibilities of studio technology (Imaginary Landscape No. 1). For Cage, however, the radio is not just a spectacular stage prop, a means of production or distribution, but an instrument in its own right. It marks the transition from composing with chance operations to a poetics of 'indeterminacy'. This applies first and foremost to Imaginary Landscape No. 4 for 12 Radios (1951), written at the same time as the famous Music of Changes. The article discusses, among other things, an audio recording of the New York premiere in May 1951, giving a few hints for musical analysis and discussing the relationship between 'indeterminacy' and an aesthetic of silence and space.

\section{Matthias Pasdzierny: „Komponierte Mediengeschichte - Bernd Alois Zimmermanns Requiem für einen jungen Dichter (1969) und das Radio“}

For Bernd Alois Zimmermann's Requiem for a Young Poet radio plays a central role in many ways. On one hand, the composition, completed in 1969, could probably not have been written and premiered without the institutional support of the Westdeutscher Rundfunk. On the other hand, the various audio tapes featured in the piece reveal Zimmermann's attempt to use the radio sound of the period between the late 1930s and 1960s as a space of memory and aesthetic experience.

\section{Janina Müller: „Der Komponist als Redner: Zur radiophonischen Stimmpolitik von Mauricio Kagels Hörspiel Der Tribun (1979)“}

In his 1979 radio play Der Tribun. Für einen politischen Redner, Marschklänge und Lautsprecher, Mauricio Kagel plays the role of a political orator exercising his demagogic skills to sway the masses. During his speech, he plays various tapes featuring parodistic military marches composed by Kagel himself, crowd noises, and other atmospheric sounds thus creating a performative space for the rehearsal. This paper seeks to contextualize Kagel's Tribun within the broader artistic field of the Neues Hörspiel ("New Radio Play") and its engagement with 
political speech. In doing so, it argues that Kagel is not merely concerned with the manipulative potential of rhetoric as such but also with the complex technical and medial frameworks through which the voices of demagogues gain powerful resonance. In its use of loudspeaker effects and cheering crowds, the radio play evokes historical associations to radio broadcasts of mass speeches of the National Socialist Party.

\section{Sara Beimdieke: „,Richtig intensiv zuhören!' - Mauricio Kagels Darstellung von Radio- hören in Rrrrrrr... Hörspiel über eine Radiophantasie (1982)“}

Mauricio Kagel is not only one of the most influential composers of the twentieth century, he is also considered as one of the key figures of the Neues Hörspiel. In Rrrrrr... Hörspiel über eine Radiophantasie (WDR, 1982), he depicts the inner thoughts of a radio listener. Through the radio play, we follow his daily routine as he has dinner, makes a phone call or reads his newspaper. The entire time music is coming from a radio in the back of his house. Kagel reflects upon the relationship between listener und music on three levels: upon the individual and his inner thoughts, upon the music in many different forms and functions as well as upon the process of listening to music. Within his radio play, Kagel explores how the attention of his listener fluctuates in relation to the music, what kind of music he prefers and how music is tied to certain memories.

\section{Susanne Kogler: „Kunstradio als ,Weltmusik’ (1996/97). Grenzüberschreitung, Kommu- nikation und Körper im Schaffen der Komponistin, Performerin und Radiokünstlerin Elisabeth Schimana"}

For several reasons Elisabeth Schimana's rich and multifarious œuvre can be considered world music: Whenever she explores innovative communication technologies, takes up scientific findings or deals with existential questions, her point of departure is a critical view of the world. From the beginning, her projects established global networks. By cooperating with Austrian's Ö1 Kunstradio she widened her sphere of influence, explored multimedia spaces and reached a wider public. By discussing her radio compositions - from early pieces created in the 1990s, such as Die Fuge, die große partitur, Obduktion and Touchless - The sensuality of music made without touching to younger works, such as Die Futuristin, or recent ones as Gebären - the paper explores how Schimana's work leads to a novel, critical understanding of world music.

\section{Anna Vermeulen: „A Critical Discourse in Sounds: Terre Thaemlitz' Trans-Sister Radio (2004)“}

Terre Thaemlitz's 2004 radio drama Trans-Sister Radio provides a compelling case study of 21st century radio art that deals with more than just aesthetic concerns. Modeled on a radio show, the 
work juxtaposes heterogeneous sonic materials which give rise to a discursive field where issues of queerness and transgenderism within radio and media, and more general issues on transgenderism in the neoliberal condition of the 2000 s are left for the listener to ponder. This article unravels this discursive field, which avoids coherent signification, but rather employs queer tactics of ambiguity and excess. When Trans-Sister Radio is read against the history of queer radio and theories on transgender cultural expression and representation, interesting perspectives on the relationship between sound and gender, and on the phenomenology of radio come to light.

Malte Kob: „Wirkung des Raumklangs bei der Produktion und Wiedergabe von Radioaufnahmen"

Rooms should have acoustic properties that characterize optimal conditions for a specific use case. A good room acoustics can either improve speech intelligibility or enhance the engulfment of listeners in a symphony hall or church. This article introduces basic concepts of rooms that provide such conditions, following a short introduction into room acoustical basics. The text further provides some examples of room acoustical optimization and methods for performers to improve music and speech projection. The article concludes with current methods for visualisation of sound field propagation and modelling of listeners' impression of music.

Zitation: Camilla Bork und Antje Tumat, "Komponieren für das Radio: Akteure, Diskurse, Praktiken”, in: Freie Beiträge zur Jahrestagung der Gesellschaft für Musikforschung 2019, hrsg. von Nina Jaeschke und Rebecca Grotjahn (= Musikwissenschaft: Aktuelle Perspektiven. Bericht über die Jahrestagung der Gesellschaft für Musikforschung 2019 in Paderborn und Detmold, Bd. 1), Detmold 2020, S. 1-5, DOI: 10.25366/2020.44. 


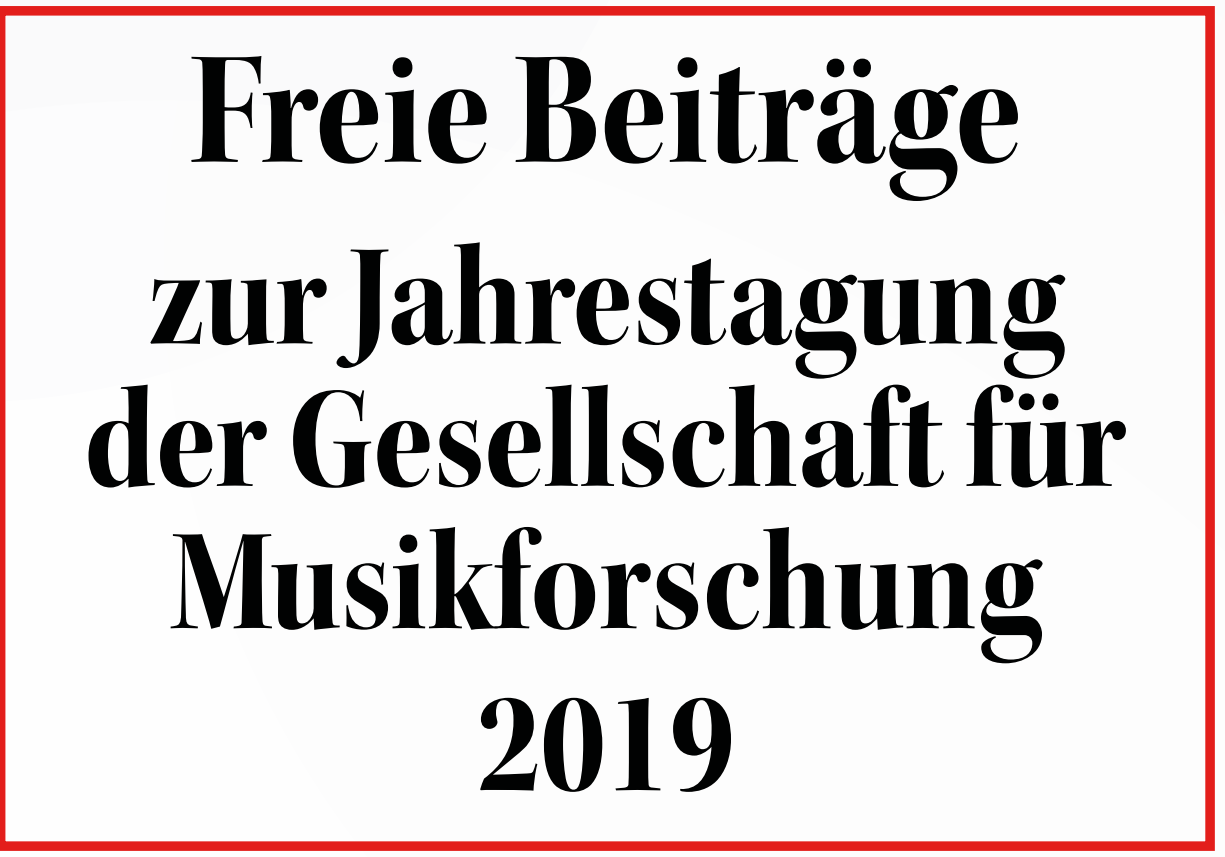

Herausgegeben von Nina Jaeschke und Rebecca Grotjahn

Musikwissenschaft: Aktuelle Perspektiven 1 
Freie Beiträge 


\section{Musikwissenschaft: Aktuelle Perspektiven}

Bericht über die Jahrestagung der Gesellschaft für Musikforschung 2019 in Paderborn und Detmold

Herausgegeben von Rebecca Grotjahn und Nina Jaeschke

Band 1 


\section{Freie Beiträge}

\section{zur Jahrestagung der Gesellschaft für Musikforschung 2019}

Herausgegeben von Nina Jaeschke und Rebecca Grotjahn

Detmold: Musikwissenschaftliches Seminar der Universität Paderborn und der Hochschule für Musik Detmold 2020 
DOI: $10.25366 / 2020.42$

Online-Version verfügbar unter der Lizenz: Urheberrecht 1.0, $<$ https://rightsstatements.org/page/InC/1.0/?language=de>

Bibliografische Information der Deutschen Nationalbibliothek

Die Deutsche Nationalbibliothek verzeichnet diese Publikation in der Deutschen Nationalbibliografie; detaillierte bibliografische Daten sind im Internet über http://dnb.d-nb.de abrufbar.

\section{Impressum}

Redaktion: Nina Jaeschke, Rebecca Grotjahn und Jonas Spieker Satz: Nina Jaeschke

(C) Musikwissenschaftliches Seminar der Universität Paderborn und der Hochschule für Musik Detmold 2020 


\section{INHALT}

Vorwort $\quad$ IX

Komponieren für das Radio: Akteure, Diskurse, Praktiken $\quad 1$

Musikwissenschaft - Feminismus - Kritik: Ein Generationenaustausch 6

\section{Stefan Alschner}

Der Wagner-Sänger Joseph Aloys Tichatschek - Vom Nachlass zum Netzwerk

\section{Alenka Barber-Kersovan}

Songs for the Goddess. Das popmusikalische Neo-Matriarchat zwischen Ethno-Beat,

erfundenen Traditionen und kommerzieller Vermarktung

Elias Berner, Julia Jaklin, Peter Provaznik, Matej Santi, Cornelia Szabó-Knotik

Musikgeschichte anders erzählen? Das Beispiel der 1970er in Österreich.

Musikhistoriographie in der Zeit der Digitalisierung

\section{Mauro Fosco Bertola}

„Ein Laut so klagevoll”. Lohengrin zwischen Richard Wagner und Salvatore Sciarrino

\section{Matthieu Cailliez}

Europäische Rezeption der Berliner Hofoper und Hofkapelle von 1842 bis 1849

\section{lacopo Cividini}

Zwischen klassischer Musikphilologie und angewandter Informatik:

Die Digitale Mozart-Edition (DME) der Stiftung Mozarteum Salzburg

\section{Marko Deisinger}

Fortschrittliche Technologie im Dienste eines Antimodernisten.

Heinrich Schenker und der österreichische Rundfunk

\section{Norbert Dubowy}

Vom Kritischen Bericht zur Kritischen Dokumentation am Beispiel der Digital-interaktiven Mozart-Edition

\section{Markus Engelhardt}

Musik zwischen Nation Building und Internationalität. Italien um 1900

\section{Maryam Haiawi}

Das Oratorium im Spannungsfeld der Konfessionen: 


\section{Judith I. Haug}

"Manch eine*r liegt, morgens noch trunken, im Rosengarten" - Rekonstruktionen

osmanischer Musikgeschichte in Gesangstextsammlungen

\section{Renate Koch}

Marcel Prawy und das erste Broadway-Musical im Österreich der Nachkriegszeit

Susanne Kogler, Julia Mair, Juliane Oberegger, Johanna Trummer

Erich Marckhl - Musikausbildung in der Steiermark nach 1945.

Brüche und Kontinuitäten

\section{Marie-Anne Kohl}

Die weinende Jury. "Geschlechtslose" Tränen bei globalen Musik-Castingshows?

\section{Fabian Kolb}

Tanztheater und filmische Ästhetik. Cineastische Einflüsse und Gestaltungsweisen in den Kompositionen für die Ballets Suédois 1920-1925

\section{Christian Lehmann}

Tempobezeichnungen von Julius Stockhausen für Die schöne Müllerin:

Ein Quellenfund

\section{Martin Link}

Signum et gens - Zur Gendersemiotik in Clara und Robert Schumanns Liederzyklus Liebesfrühling

\section{Livio Marcaletti}

„Strafspiel" und satirische Stilmittel in musikdramatischen Gattungen des frühen 18. Jahrhunderts

\section{Tobias Marx, Martin Lissner}

Thüringer Musikszene - Jugendmusikredaktionen als außerschulische musikbezogene Bildungskontexte

\section{Maho Naito}

Die Parallelität der Entstehungsprozesse der ersten beiden Symphonien Gustav

Mahlers: Instrumentation, Revision und Dirigierpraxis

\section{Elisa Novara}

Eine Schumann-Werkstatt? Zur Übertragbarkeit der Methoden vom Projekt 
Theodora Oancea, Joachim Pollmann, Jonas Spieker

Kollaborateure - Involvierte - Profiteure. Erarbeitung eines Online-Lexikons zur

Musik in der NS-Zeit

\section{Kiron Patka}

„Ich wollte eigentlich Sängerin werden." Berufsselbstbilder von Tontechniker*innen im Radio

\section{Siegwart Reichwald}

Die Leiden der jungen Clara: Das Klaviertrio Opus 17 als Ausdruck einer Neu-

Romantikerin

\section{Elisa Ringendahl}

Lied versus Oper - Pole musikalischer Gattungen bei Oscar Bie

\section{Benedikt Schubert}

Struktur und Exegese. Über Eigentümlichkeiten in der Arie "Des Vaters Stimme ließ sich hören" (BWV 7/4)

Uwe Seifert, Sebastian Klaßmann, Timo Varelmann, Nils Dahmen

Computational Thinking in der Musikwissenschaft: Jupyter Notebook als Umgebung

für Lehre und Forschung

\section{Yusuke Takamatsu}

Synthese als Modus der Prozessualität bei Schubert:

Sein spezifisches Wiederholungsprinzip im langsamen Satz

\section{Daniel Tiemeyer}

Johann Nepomuk Hummels Sonate in fis-Moll Op. 81 - Studien zu Entstehungs-

hintergrund, Rezeption und formalerStruktur

\section{Andrea van der Smissen}

Musikalische Innovation im Umfeld der Moderne und historischen Avantgarde in Ungarn

\section{Tim Ziemer, Holger Schultheis}

Psychoakustische Sonifikation zur Navigation in bildgeführter Chirurgie

\section{Magdalena Zorn}

Musik mit dem Radio hören: Über den Begriff der musikalischen Aufführung 
Gabriele Buschmeier in memoriam 


\section{Vorwort}

Die vorliegenden Bände dokumentieren die Jahrestagung der Gesellschaft für Musikforschung 2019. In den dreieinhalb Tagen vom 23. bis zum 26. September 2019 wurden in Paderborn und Detmold nicht weniger als 185 Beiträge präsentiert, verteilt auf diverse Symposien, Round tables, Freie Sektionen und Postersessions. Sie alle auf einen Nenner bringen zu wollen, ist ein Ding der Unmöglichkeit - und das ist gut so, ist es doch Ziel der Jahrestagungen, die große Vielfalt der Themen und Methoden des Faches Musikwissenschaft abzubilden. Um die thematische Vielfalt der freien Referate angemessen abbilden zu können und gleichzeitig den inhaltlichen Schwerpunkten der beiden hier publizierten Hauptsymposien ausreichend Raum bieten zu können, erscheinen diese in drei Bänden.

„Musikwissenschaft: Aktuelle Perspektiven": Der Titel der kleinen Reihe ist keine Verlegenheitslösung. Musikwissenschaft im Kontext der Digital Humanities; Musikwissenschaft und Feminismus; Musik und Medien; Musikalische Interpretation - schon die Felder, die von den vier Hauptsymposien bespielt wurden, wären noch vor wenigen Jahrzehnten allenfalls an der Peripherie das Faches zu finden gewesen. Sie entsprechen Arbeitsschwerpunkten der Lehrenden am Musikwissenschaftlichen Seminar der Universität Paderborn und der Hochschule für Musik Detmold, das die Tagung ausrichtete. Zugleich nehmen sie Bezug auf aktuelle Ereignisse und Entwicklungen. So erwuchs das von Andreas Münzmay und Joachim Veit organisierte Symposium „Brückenschläge - Informatik und Musikwissenschaft im Dialog" unmittelbar aus den Erfahrungen im Virtuellen Forschungsverbund Edirom (ViFE) und im fakultäten- und hochschulübergreifenden Zentrum Musik-Edition-Medien (ZenMEM). Der 200. Geburtstag von Clara Wieck/Schumann war der Anlass für das von Rebecca Grotjahn geleitete Symposium „Die Begleiterin - Clara Schumann, Lied und Liedinterpretation", das in enger Kooperation mit der Hochschule für Musik Detmold durchgeführt wurde. Das Hauptsymposium „Brückenschläge" wird in einem separaten Band publiziert (Bd. 3 der vorliegenden Reihe). Im Rahmen dieses Symposiums führte die von Stefanie Acquavella-Rauch geleitete Fachgruppe Digitale Musikwissenschaft eine Posterpräsentation durch, die von den Beiträger*innen erfreulicherweise zu kürzeren Texten umgearbeitet wurden, sodass sie hier ebenfalls, zusammen mit den Postern,

publiziert werden können. Hinzu kommen einige Beiträge, die bereits bei der Jahrestagung 2018 in Osnabrück präsentiert wurden. Auch das Hauptsymposium "Die Begleiterin" wird in einem eigenen Band (Bd. 2) publiziert. Die Beiträge zu den beiden anderen Hauptsymposien hingegen werden an anderen Orten veröffentlicht; in Band 1 („Freie Beiträge zur Jahrestagung der Gesellschaft für Musikforschung 2019") der vorliegenden Publikation finden sich jedoch Einführungen und Abstracts. Das Symposium „Komponieren für das Radio" unter Leitung von Antje Tumat und Camilla Bork (Katholieke Universiteit Leuven) behandelte Einflüsse des Mediums auf Kompositionsprozesse sowie durch radiophone Kompositionen bzw. radiophonen Klang ausgelöste Diskurse. Sarah Schauberger und Cornelia Bartsch (Universität Oldenburg) nahmen das 25-jährige Jubiläum der Fachgruppe Frauen- und Genderstudien zum Anlass für einen Generationenaustausch zum Thema "Musikwissenschaft - Feminismus - Kritik": Was wa- 
ren vor einem Vierteljahrhundert Inhalte und Aufgaben einer feministischen Musikwissenschaft und wie kann sich diese heute positionieren?

Bewusst haben wir im Tagungsbericht auf inhaltliche Eingriffe in die Beiträge verzichtet. ${ }^{1}$ Das gilt besonders für die Freien Referate: Es galt, den Charakter der Jahrestagung als Forum für ,freie', d. h. innovative und auch experimentelle Gedanken zu wahren. Einige Kolleg*innen, die die Tagung mit Vorträgen und Posterpräsentationen bereichert hatten, haben sich gegen eine Publikation im vorliegenden Band entschieden - sei es, weil sie eine Möglichkeit fanden, ihre Beiträge in einem inhaltlich passenderen Rahmen zu veröffentlichen, sei es, weil ihre Überlegungen in ihre entstehenden Qualifikationsschriften fließen sollen, oder sei es, weil sie von den Autor*innen in der vorgetragenen Form zunächst verworfen wurden. Auch damit erfüllt eine Freie-Referate-Sektion ihren Zweck: Die Diskussionen mit der versammelten Fach-Öffentlichkeit sollen dabei helfen, Gedanken weiterzuentwickeln und zu verändern. In diesem Sinne sei allen Beteiligten - den Autor*innen, den nichtpublizierenden Referent*innen und den Mit-Diskutant*innen - ganz herzlich gedankt für ihr Mitwirken bei der Tagung.

Unser herzlicher Dank gilt einer Reihe weiterer Personen, die zum Gelingen dieser drei Bände beigetragen haben. Hier ist besonders Jonas Spieker zu nennen, der uns tatkräftig bei der Redaktion geholfen hat. Andrea Hammes (SLUB Dresden) sei herzlich für die Aufnahme unseres Bandes auf musiconn.publish gedankt - wir freuen uns, damit unsererseits zur Etablierung dieser innovativen Publikationsplattform beizutragen.

Erneut möchten wir an dieser Stelle allen Menschen danken, die uns bei der Organisation, Ausrichtung und Finanzierung der Tagung selbst unterstützt haben: der Präsidentin der Universität Paderborn, Prof. Dr. Birgitt Riegraf, dem Rektor der Hochschule für Musik Detmold, Prof. Dr. Thomas Grosse, den Kolleginnen und Kollegen der beiden beteiligten Hochschulen, dem Vorstand der Gesellschaft für Musikforschung, der Universitätsgesellschaft Paderborn und allen Sponsoren. Besonders dankbar sind wir den Mitarbeiter*innen und den studentischen bzw. wissenschaftlichen Hilfskräften des Musikwissenschaftlichen Seminars, die bei der Vorbereitung und Ausrichtung der Tagung immensen Einsatz zeigten - stellvertretend sei an dieser Stelle Johanna Imm erwähnt, die zusammen mit Nina Jaeschke das Herz des Organisationsteams bildete.

Wir widmen diese Reihe Dr. Gabriele Buschmeier, dem langjährigen Vorstandsmitglied der Gesellschaft für Musikforschung, die kurz vor der Publikation dieses Bandes unerwartet verstarb.

Detmold, im September 2020

Rebecca Grotjahn und Nina Jaeschke

Zitation: Rebecca Grotjahn und Nina Jaeschke, „Vorwort”, in: Freie Beiträge zur Jahrestagung der Gesellschaft für Musikforschung 2019, hrsg. von Nina Jaeschke und Rebecca Grotjahn (= Musikwissenschaft: Aktuelle Perspektiven. Bericht über die Jahrestagung der Gesellschaft für Musikforschung 2019 in Paderborn und Detmold, Bd. 1), Detmold 2020, S. IX-X, DOI: 10.25366/2020.43.

1 Freigestellt war den Autor*innen auch, ob sie sich für eine gendersensible Sprache entscheiden bzw. welche Form des Genderns sie bevorzugen. 